\title{
The Discussion of the Possibility and Development pattern for Constructing the Public Security Management System based on Big Data
}

\author{
XueHeng Gong ${ }^{1, a}$, Jing Sun ${ }^{1, b}$ and YiPing Zhang ${ }^{2, c}$ \\ ${ }^{1}$ Department of Public Security, Peoples Public Security University of China, BeiJing, China \\ ${ }^{2}$ Department of Science and Technology, Yunnan Police Officer Academy, Kunming, China \\ a1501421123@qq.com, bgoodwill331@126.commail, 'Zhangyiping_001@163.com \\ Corresponding Author: YiPing Zhang \\ Co_Author E-mail: Zhangyiping_001@163.com
}

\begin{abstract}
Keywords: Big data analysis, Police information system, Public Security system, Hadoop.
\end{abstract}
\begin{abstract}
The key purpose of this paper aims to discuss the possibility of constructing the public information system based on the Big data. Especially, the public security information system is chosen as the objective for details. Then the significance and difficulties which we will face when the corresponding big data analysis system is constructed are discussed. Meanwhile, both two aspects are extended to obtain the ideal of construction pattern which should be considered. The final discussion comes to the conclusion that the structure of the future extended big data system for police application is pictured, which give the basis for construction specific big data police application system. The analysis and discussion indicate that the construction of public security system based on Big data is possible and the corresponding technology is mature.
\end{abstract}

\section{Introduction}

In Mid-19th century, the steam engine opened the time called " the first industrial revolution" , which take the human world into the path of rapid development. On the early 20th century, the utilization of electricity promoted the Second Industrial Revolution. At the same time, the natural sciences, especially the development of mathematics and statistics, the fact that the human society can be described by a mathematical model come true. Meanwhile, the development of technology has also brought social and cultural developing rapidly, with forming a large-scale "knowledge explosion. Based on this fact, the rapid development of modern university education is improving the level of human culture fundamentally. At the latest 20th century, the development of modern computers and electronics have brought new historical leap in human society. In this time, the word "Digital" became a human familiar vocabulary. Then, Internet has become the main platform for data exchange, which makes the distance between countries being shortened. Furthermore, culture, information, intelligence and even passing messages has no longer been subject to geographical factors, which greatly reduce the human speed accessing to information. Therefore, the word "Data" has become a strategic resource for governments attaching great importance[1]. For example, the "information superhighway" proposed by USA government aims to improve the development of the national strategic level.

However, when the wheel of history are role into the 21 st century, human society is facing a new opportunity, perhaps, this is a new opportunity to say --- "big data". Narrowly speaking, the term big data refers to the mass storage for data. Oppositely, the Big Data implies the information harvesting by using mathematics, theoretical statistics and the high-speed computer from large databases[2]. By analyzing data patterns hiding in data, he possibility one thing will happen can be predicted. Actually, on the first 10 years of the 21 st century, human society is the era of data explosion with the popularity of mobile computing platforms, the birth of high-performance computing equipment, the timing of the development of modern statistics, information management platform for big data research in an era ripe . Currently, there are many companies use "big data," it is the opportunity to expand their 
business by obtaining complete profit model transformation and to bring amazing profit growth[3]. Such as the famous Amazon, Facebook and so on.

On the other hand, the big data also play more role for the police information construction[4,5]. For example, the "three-building" project, it relies on the development of the technology. One of the face of the impact of big data era is the technology. Meanwhile, both the challenge and the opportunity exist. The current status of public security information technology, briefly speaking, can only achieve "digital" level, which is far from the "data" of the hierarchy. Despite the utilization of the universal application of the office automation system, the "police mechanized" platform, large information systems, information management systems can only help the easy analysis for the public security information. Furthermore the current management information system (MIS or Expert Decision System) can only facilitate the daily police work, for data management is only completed junior record store. The more complex systems, such as large information systems, data can be obtained by only some simple pattern recognition, or by making some simple decisions. The potential value of the data for the current public security information system is not fully utilized. In the era of big data, data mining methods is used to excavate the information and the results are translated to the expert system[6]. In other words, decision theory system used in the digital age is not suitable for large data mining information. To build large data public security information system, we need to study the new data modeling, analysis, forecasting strategy mode. The study of these contents relies on having a Master Model for guidance. Therefore, Public Security Information construction under the Big Data era is important for future work. In this paper, the possibility for constructing the big data application for police security is discussed.

\section{The significance of the construction of Big data for police application}

Big data processing mode is important for speeding up the construction of public security information. The context of data using a large data analysis and decision-making ability and the public security organs can be more convenient to access to the intelligence sources, it can also be completed quickly merge need to use the clues. At the same time, large data system can also help a clearer understanding of the public security organs trends humanistic nature of specific regions. Therefore, in terms of many aspects of public security work, public security information technology can build large data directly which is related to the future of public security work carried out efficiently. Furthermore, the public security organ of intelligence information can also improve the access speed of trailing information.

In details, the significance lies in the following aspects:

Firstly, the basic functions of large data systems can provide quick access to information from massive data distributed. In history, especially in 19th century, the Rothschilds took advantage to build their own rapid intelligence network in advance to detect the news of the defeat of Napoleon, which was able to earn in the London financial markets pours [1]. In the 20th century, the Amazon use their textbook to grasp of user record information and to recommend books to readers completed the creative work and ultimately success. Of course, the big data not only can provide business services. For public security work, big data applications can also play a role to analysis, decision-making by using a large data. Investigating techniques will greatly expand the public security organs.

Secondly, relying on large data analysis and decision-making, the public security organs to quickly analyze clues, locked the suspect. From the information theory point of view, investigation, interrogation, investigation and other works conducted by the public security organs in theory is to make information (uncertainty) criminal event contains a minimum. It has been put into the public security information system used to some extent and to accelerate the realization of the above process. However, due to the small size of the dataset, we can rely on clues intersection method to look for clues. This approach by seeking event, then the intersection of character attributes and the accurate information will determine clues. However, finding the cross point is not an easy thing in the complex set of clues. For large data, due to the large amount of data, it often does not require precise intersection analysis as a basis for relations. 


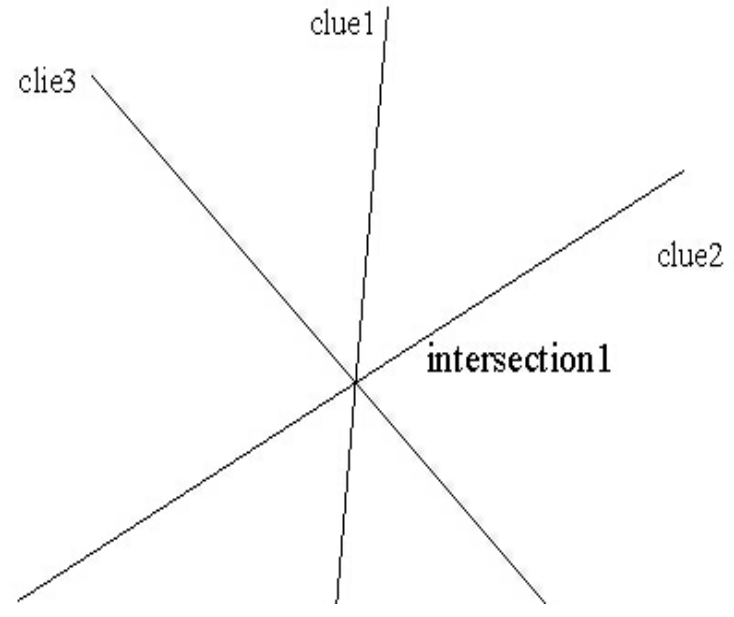

(a) Traditional analysis based on clues

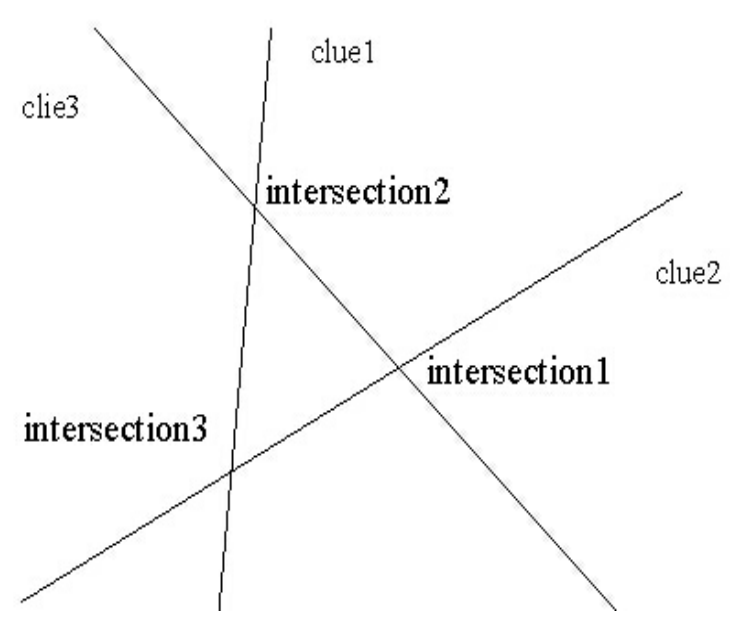

(b) Analysis clues based on Big data

Fig.1 The sign of two analysis methods based on traditional way and big data respectively

From figure 1, the more clues clues space are constituted. In order to obtain useful information cross, it must complete multi-threaded with multi-line indication line level crossings. On the other hand, however, data from multiple clues of the cube space plane may be in the form of the intersection to form a support plane estimate estimate. Thus such a way that the estimated gain clues which is better than the era when small data existed can be more easier. At the same time, with an estimated decision-making capacity, it often can get more than a small data sets to obtain more useful clues message. It also implies that the large public security information system should take more advantage of the current information system.

Finally, the big data can be more truly reflect the relationship between people and the analyze trends of Social Relations particular environment. Currently, the public security information system based on the relational model uses a two-dimensional table structure (relational database using a two-dimensional table describes the data association) to describe the community of people attribute and relationship with each other. The results described in this way brings a loss of information associated with the space, and it relies on a large-scale relational database for querying processing statements in order to obtain the required information. In contrast, large data management results are described by using a spatial grid which enable the multiple spatial pattern (multiple threads get way) to describe the information obtained. For example, for constructing the analysis model to obtain the citizens' basic information, such as letters and other messages, the interpersonal relationships extending from the relationship diagrams can make predictions for the region's population with more activity. Meanwhile, it is important to avoid mass incidents.

\section{Difficulties}

Actually, there are many difficulties during the construction of the public security information system based on big data. They are listed as follows:

(A) the current data pattern using all types of public security information system is not uniform. Current data storage in the system of public security information is not discarded. Therefore, when building large data systems, we need to use the system in an integrated manner to integrate the current system resources. However, the multi-mode data integration is hard to achieve.

(B) The types of information are various, it implies that it is difficult to obtain the useful information from multi-source data directly. These information include text, audio, image, video, and network data and so on. Meanwhile, With the development of science, the more type of information will take part in the estimation process. However, the approach to tackle these muti- data can not suit for the requirement. 
However, anything new development will face difficulties. Construction of large data information system security is no exception. Considering its great practical value for the difficulties, we propose a design pattern to explore the possibility corresponding to Promote Public Security Information System Construction data.

\section{Construction model}

Construction of large data public security information technology is necessary to give full play to large data fast, the strong analytical skills and the diversification of sources of intelligence information features, but it should also consider the full use of existing resources, good data systems integration and the maximize cost savings. To solve these problems, when building large data public security information technology, the following three aspects should be considered.

(A) New statistical algorithms and faster data acquisition

Mathematical and statistical algorithms are used to analysis the large data quickly to extract valuable information simply. It requires the use of high efficiency, high reliability of statistical algorithms. Actually, the modern analysis technology such as SVM and the minimum description length[7,8], can be suggested to help the analysis process when system builders need to consider the maintainability and scalability.

(B) the gradual completion of data integration

To save costs, the system used at this stage need to continue to utilization. Data used in the existing system can be divided into two categories, one is the data which is already stored in the system, and the other is the current newly acquired data. Historical data, follow the "side use, while integration" principle Tai gradually integrate data systems integration can be taken when using low frequency data before transplantation, the use of high frequency data gradual migration to finalize all data migration conducted.

For the newly acquired data, it should be directed in accordance with the requirements of large data system data structure data organization, and it also needs the additional data to complete the organizational form of a current system which can be recognized. Thus, the new data on the workload should be greater.

(C) the characteristics of the data with a central cluster data integration

Large data systems with distributed data should have the parallel processing ability. In the big data information system security system, it is designed with considerations this feature. In the system design, it can be considered by the central cluster data unit where the underlying data structure design interface for data systems (on macro-organization of data definition), and the unit of data in a distributed, according to the actual situation in the region, at the macro basic data structure of the interface between their respective characteristics of the data. With this design approach, characteristic data having a small and flexible features, but can fully reflect the details of a particular region. The central cluster of data can optionally use the characteristics of the data, it has completed a comprehensive analysis of the maximum degree of data applications.

\section{Conclusion}

Big data public security information technology is the future trend of public security information technology, is to create the police, the data of the police, intelligence-based policing and information technology. Give full play to the strength under the era of big data data information, to tap the high-value intelligence data trail behind large, for speeding up the public security law enforcement capacity building is important. With the further popularization of the concept of big data, combined with public security work and modern technology will become a powerful weapon in the hands of police officers. The full development of big data of public security information technology to improve enforcement capacity in modern science and technology background and the background of large data lay the foundation for improving the level of public security organs of law enforcement, this is the most fundamental purpose of large data Public Security Information. 


\section{Acknowledgements}

This work is supported by Natural Science Foundation of Yunnan Province under Grant 2014FD037. Meanwhile, the author want to appreciate the professor Zhang Yi-Ping for inspiring this ideal.

\section{References}

[1] [1] Viktor. M. Schönberger, Kenneth Cukier, A Revolution That Will Transform How We Live, Work, and Think, Houghton Mifflin Harcourt, 2013.

[2] Asur, Sitaram and Bernardo A.Huberman, "Predicting the Future with Social Media.", Proceedings of the $2010 \mathrm{IEEE} / \mathrm{WIC} / \mathrm{ACM}$ International Conference on Web Intelligence and Intelligent Agent Technology, 2010, pp.492-499.

[3] Lee,YH.,Hsieh,Y, Adding Innovation Diffusion Theory to the Technology Acceptance Model: porting Employees' Intentions to use E-Learning Systems[J] . Educational Technology\& Society,14 (4), 2011:124 -137

[4] Hsin-Chang Yang, hung-Hong Lee. 2005. A text mining approach for automatic construction of hypertexts. Expert Systems with Applications, 9:723-734.

[5] Schweitzer F, Fagiolo G, Sornette D, Vega-Redondo F, Vespignani A, et al. (2009) Economic Networks: The New Challenges. Science 325: 422-425.

[6] Bapler.P\&Murdoch, Academic Analytics on Data Mining in Higher Education. Intemational Jounlal for the Scholarship of Teaching and Leanling, 4(2),2013.

[7] Min Chen etc, Context Quantization Under the Minimum Increment of the Adaptive Code Length[C], proceeding of the information Technology IEEE, 2013, HongKong : 9-12.

[8] Min Chen, Jianhua Chen, Affinity Propagation for the Context Quantization[J], Advanced Materials Research, vol(791), 2013: 1533-1536. 